\title{
Scoring imprecise credences: A mildly immodest proposal
}

\author{
CONOR MAYO-WiLson AND GREGORY WHEELER
}

Forthcoming in Philosophy and Phenomenological Research

Jim Joyce argues for two amendments to probabilism. The first is the doctrine that credences are rational, or not, in virtue of their accuracy or "closeness to the truth" (1998). The second is a shift from a numerically precise model of belief to an imprecise model represented by a set of probability functions (2010). We argue that both amendments cannot be satisfied simultaneously. To do so, we employ a (slightlygeneralized) impossibility theorem of Seidenfeld, Schervish, and Kadane (2012), who show that there is no strictly proper scoring rule for imprecise probabilities.

The question then is what should give way. Joyce, who is well aware of this no-go result, thinks that a quantifiability constraint on epistemic accuracy should be relaxed to accommodate imprecision. ${ }^{1}$ We argue instead that another Joycean assumptioncalled strict immodesty - should be rejected, and we prove a representation theorem that characterizes all "mildly" immodest measures of inaccuracy.

\section{ACCURACY}

Why should rational degrees of belief abide by the axioms of probability? The traditional answer, advanced by Ramsey (1926) and de Finetti (1937), is that agents whose partial beliefs fail to do so expose themselves to a risk of sure loss. Despite its ingenuity, this so-called "Dutch Book" argument has failed to impress some epistemologists (Christensen 1996; Joyce 1998), who argue that probabilism should be given a purely epistemic defense. Avoiding sure loss, they argue, is a pragmatic goal and cannot undergird epistemic norms.

De Finetti later developed a second argument for probabilism that comes closer to meeting the criticisms of epistemologists (de Finetti 1974). On de Finetti's second scheme, an agent announces a real number that represents how strongly she believes a proposition, and she does so on the understanding that she will be penalized by how far her announcement diverges from the truth-value (zero or one) of the proposition. The penalty, which is calculated via a scoring rule, is extracted by an experimenter in some currency of value to the agent. De Finetti argued that, as long as the scoring rule possesses certain mathematical properties, a rational agent's degrees of belief ought to satisfy the probability axioms. Otherwise, the agent can adopt probabilistic credences that decrease her penalty whatever the truth may be.

\footnotetext{
${ }^{1}$ In personal conversation, Richard Pettigrew and Hannes Leitgeb have advocated doubling-down on accuracy and view this result as all the more reason to reject imprecision.
} 
Unfortunately, for many epistemologists, de Finetti's second argument suffers from the same difficulty as the first: it employs a pragmatic criterion-minimizing loss in some currency-in order to justify an epistemic norm. Eliminating this pragmatic scale was the motivation for Jim Joyce's program (1998). Although Joyce employs de Finetti's scoring-rule framework, his philosophical motivations differs substantially. Joyce argues that scoring rules can be used to measure "gradational accuracy" of an agent's belief state and that accuracy is a purely epistemic good. So long as measures of gradational accuracy possess the same mathematical properties as de Finetti's scoring rules, one can employ de Finetti-like theorems to provide a non-pragmatic defense of probabilism. Joyce's philosophical insight (and challenge), therefore, was to defend the claim that measures of inaccuracy satisfy similar enough axioms to get a de Finetti-like theorem off the ground. ${ }^{2}$

To state the axioms that Joyce and other accuracy-first epistemologists endorse, let $\varphi$ denote your favorite contingent proposition, ${ }^{3}$ such as "The coin will land heads on the next toss", and let $B$ be the set of possible belief states one might have with respect to $\varphi$. For the moment, we will remain agnostic about the proper way to represent belief. For example, if beliefs are numerically representable, members of $B$ might be probability functions. Alternatively, $B$ might contain intervals of numbers if beliefs are indeterminate or "imprecise." Or $B$ might contain some other type of object altogether (e.g., sets of propositions, ranking functions, etc.). For now all we assume, which is standard among epistemologists, is that there is some way of quantifying how similar two belief states are.

What criteria should a measure of gradational accuracy satisfy? All accuracyfirsters endorse at least three. First, any measure of inaccuracy must be extensional (Joyce 1998, p. 591). Extensionality requires that inaccuracy is a function exclusively of one's belief state and the truth-value of the proposition of interest. Thus, we will write $I(b, \omega)$ to denote the inaccuracy of the belief $b$ if the truth-value of $\varphi$ is $\omega$. Joyce argues that extensionality is a core thesis of accuracy-first epistemology and that most objections to it conflate the "epistemic utility" of a belief, which might incorporate facts about the simplicity, fruitfulness, or some other value, with accuracy, which only measures the distance of the belief from the truth:

... gradational accuracy is supposed to be the analogue of truth for partial beliefs. Just as the accuracy of a full belief is a function of its attitudinal "valence" (accept/reject/suspend judgment) and its truth-value, so too the accuracy of a partial belief should be a function of its "valence" (degree) and truth-value (Joyce 1998, p. 592).

Second, all accuracy-firsters endorse a continuity postulate, which says that if two belief states $b$ and $b^{\prime}$ are sufficiently similar, then their degrees of inaccuracy are also similar. For example, if Jules' and Jim's beliefs are representable by probabilities and

\footnotetext{
2Joyce's program has inspired a number of philosophers (Leitgeb and Pettigrew 2010; D' Agostino and Sinigaglia 2010) to search for epistemic criteria that determine a narrower class of functions measuring "gradational accuracy."

${ }^{3}$ Joyce and others typically evaluate accuracy of a belief state with respect to a finite set of propositions. We restrict ourselves to one proposition for simplicity.
} 
both believe some proposition strongly — say, to degree .99 and .991 , respectivelythen their beliefs should be similarly accurate or inaccurate, depending upon whether the proposition is true or not. Formally, accuracy-firsters assume the function $I(b, \omega)$ is a continuous function of $b$ for any fixed truth-value $\omega$. In defending a postulate strictly stronger than continuity, Joyce argues that the postulate "should be uncontroversial given that gradational accuracy is supposed to be a matter of "closeness to the truth'" (ibid, p. 591). Leitgeb and Pettigrew motivate continuity by claiming that if inaccuracy "were discontinuous as a function of [one's credences], an agent's accuracy could improve or deteriorate dramatically by an arbitrarily small change to her degree of credence" (2010, p. 226).

Before turning to the third postulate, a clarification. Thus far we have remained agnostic about the correct way to represent belief, but when defending the previous two constraints on inaccuracy measures accuracy-firsters typically assume that beliefs are representable by real numbers. Even so, what the above passages show is that the arguments accuracy-firsters offer do not mention the way in which belief is represented. Hence, if the above arguments are valid when belief is numerically representable, then they are also valid when beliefs are represented by indeterminate probabilities or any set of possible belief states-so long as distances between beliefs can be quantified. In contrast, the most plausible arguments for the third postulate do seem to rely at least implicitly on the way in which belief is represented. Namely, it is commonly assumed by accuracy-firsters that belief is represented by a real number between zero and one, inclusive.

The third postulate is that inaccuracy can be numerically quantified by a nonnegative real number. Leitgeb and Pettigrew motivate this postulate as follows:

A rational agent's degree of belief for a proposition is nothing but the agent's best possible estimate or 'simulation' of the truth value of that proposition, given her present epistemic situation. Since truth and falsity [are] represented by real numbers, too, degrees of belief and truth values are comparable - they occupy the same quantitative or geometrical scale. So, for example, assigning a degree of belief 1 to a proposition A would mean that the agent believes that $\mathrm{A}$ is true rather than false, since the degree of belief 1 is closer-in fact, identical-to the real number 1 that represents truth than it is to the real number 0 representing falsity. In this way, closeness of a degree of belief to the truth can be measured (Leitgeb and Pettigrew 2010, pp. 211-2).

In other words, Leitgeb and Pettigrew claim that because both degrees of belief and truth values are numerically representable, so are their degrees of closeness. We think this argument is problematic (Mayo-Wilson and Wheeler, ms), but nonetheless, we endorse a restricted version of Quantifiability for reasons discussed in Section 4.

Armed with a measure of inaccuracy, Joyceans then argue for probabilism and other epistemic norms, such as conditionalization. However, axiomatic constraints on measures of accuracy alone cannot justify normative epistemological theses: one also needs rationality postulates that dictate how a measure of accuracy constrains the set of rational credences. Four are relevant for our purposes. 
The first rationality condition is admissibility (Fishburn 1970; Sen 1971):

A1. Admissibility: Let $b, c$, and $d$ be three (not necessarily distinct) belief states, and suppose that $d$ is at least as accurate as $c$ whatever the truth. If an agent's belief state is $b$ and the set of rational belief states $R_{b}$ from her perspective contains $c$, then it also contains $d$.

Admissibility ${ }^{4}$ is less frequently discussed in the philosophical literature than dominance (Savage 1972; de Finetti 1981), which is often used in place of admissibility:

A2. Dominance: If $d$ is strictly less accurate than $c$ whatever the truth, then $d$ is not a rational belief state, i.e., regardless of one's beliefs $b$, the set of rational beliefs $R_{b}$ from one's perspective does not contain $d$.

Although Admissibility and Dominance seem intuitively plausible, both principles are suspect unless one is careful about the interpretation of $R_{b}$. Consider Admissibility first, and suppose Bill's and Carole's beliefs are always equally accurate, regardless of the truth. Then Admissibility entails that Bill's beliefs are rational if Carole's are. That's obviously false if epistemic rationality depends on something other than accuracy. For if Carole's beliefs are well-warranted by the evidence and Bill's are not, then Bill might be irrational even if his beliefs will turn out to be as accurate as Carole's. Although their argument is considerably more subtle, Easwaran and Fitelson (2012) likewise argue that Dominance and evidential considerations can conflict. ${ }^{5}$

For our purposes, these concerns can be avoided by stipulating that we interpret $R_{b}$ to be those credences that are rational on the basis of accuracy-related considerations alone from the perspective of an agent in belief state $b$. We consider this interpretation for two reasons. First, some accuracy-first epistemologists hold the view that accuracy is the only epistemic good (Pettigrew 2013). Second, and more importantly, evidence is not always plentiful, and consequently, it is necessary to investigate epistemic norms in the absence of significant evidence. Interpreting $R_{b}$ in this limited way helps us do this.

The third rationality postulate is Immodesty. Modest individuals often undersell their own accomplishments. By analogy, a credence is modest if it entails that some other credence is strictly more accurate. Thus, immodesty is the principle that a rational individual ought not find her own belief state to be irrational. If $R_{b} \subseteq B$ is the set of belief states that are rational from the perspective of someone whose belief state is represented by $b$, then Immodesty is the condition that $b \in R_{b}$.

Channeling Gibbard (2007), Joyce argues for Immodesty as follows:

Modest credences, it can be argued, are epistemically defective because they undermine their own adoption and use. Recall that a person whose

\footnotetext{
${ }^{4}$ Joyce (2009) uses "admissibility" to refer to a different principle, namely, the principle that one ought not adopt a credence $b$ if there is some credence $c$ that (i) is at least as accurate as $b$ whatever the truth and (ii) is strictly more accurate for some truth assignments to the propositions of interest. Joyce's principle, we think, ought to be called "weak dominance" in order to be consistent with typical decision-theoretic terminology.

${ }^{5}$ See (Joyce 2009) for a response.
} 
credences obey the laws of probability is committed to using the expectations derived from her credences to make estimates. These expected values represent her best judgments about the actual values of quantities. If, relative to a person's own credences, some alternative system of beliefs has a lower expected epistemic disutility, then, by her own estimation, that system is preferable from the epistemic perspective. This puts her in an untenable doxastic situation. She has a prima facie epistemic reason, grounded in her beliefs, to think that she should not be relying on those very beliefs. This is a probabilistic version of Moore's paradox. Just as a rational person cannot fully believe 'X but I don't believe $X$,' so a person cannot rationally hold a set of credences that require her to estimate that some other set has higher epistemic utility. The modest person is always in this pathological position: her beliefs undermine themselves (Joyce 2009, p. 277).

The final rationality condition, which we will call Strict immodesty, is not endorsed by all accuracy-firsters, but it is explicitly defended by Joyce (2009) and a number of other epistemologists (Oddie 1997; Greaves and Wallace 2006; Gibbard 2007). Strict immodesty asserts that a rational individual ought to find only her belief state to be rational; that is, $R_{b}=\{b\}$.

It is essential that both Immodesty and Strict Immodesty describe a rational agent's beliefs. Someone with irrational beliefs (e.g., who endorses a contradiction) might find other beliefs to be rational, and she might find her own to be defective. This would not undermine either Immodesty or Strict Immodesty. So although our formal statement of both principles quantify over all belief states $b$, it is important that our arguments depend only on the assumption that probabilistic belief states can be rational, which (Joyce 2009)[p. 279] endorses. Further, because what it is rational to believe depends upon how one measures inaccuracy, both Immodesty and Strict Immodesty implicitly rule out particular measures of inaccuracy. For instance, neither is compatible with a measure of inaccuracy that entails that one particular credence $b$ is strictly more accurate than all others, for then someone with different probabilistic credences could not consider herself rational, contradicting our assumption. ${ }^{6}$

Strict Immodesty entails a principle similar ${ }^{7}$ to the so-called uniqueness thesis, which asserts there is a uniquely rational belief state that is warranted by one's evidence (White 2005; Feldman 2011; Kelly 2002). Why? Strict immodesty entails that, from a rational agent's perspective, accuracy-related considerations determine a uniquely rational belief state, and being appropriately responsive to one's evidence can only narrow the set of rational beliefs.

\footnotetext{
${ }^{6}$ Thanks to Jason Konek for this clarification. Formally, what this means is that $R$ ought to be a function both of an inaccuracy measure $I$ and a belief state $b$, and Immodesty ought to be understood as the principle that $b \in R(b, I)$ for all $b$. So Immodesty is a feature of the pair $\langle I, R\rangle$, not just $R$.

${ }^{7}$ Importantly, Strict Immodesty does not entail the uniqueness thesis, and Joyce endorses the former but not the latter. One may endorse Strict Immodesty and accept that there are many credences that are rational given one's evidence. What Strict Immodesty says is that it is irrational to adopt some credence $b$ and to believe some other credence $c$ is more accurate. In contrast, the uniqueness thesis says that there is a unique rational credence compatible with $e$. Thanks to an anonymous referee for making this point clear to us.
} 
The intuition motivating the uniqueness thesis is the following. Suppose the uniqueness thesis were false. Then there is some rational individual who has belief state $b$, but also recognizes that some other belief state $c$ is rational. For example, suppose $B$ is a set of propositions representing an agent's "full" beliefs. If $c$ is not equal to $b$, then there is some proposition in $b$ that is not in $c$ or vice versa. Without loss of generality, assume that $c$ contains some proposition $\psi$ that $b$ does not. In this case, the individual believes that $\psi$ is warranted by the available evidence (as she believes $c$ is a rational belief state), but she does not endorse $\psi$ herself (as $\psi$ is not in $b$ ). For many, this seems like a strange position. Either belief in a proposition is warranted by evidence or it is not. In the former case, one should believe it; in the latter, one should not.

Although the uniqueness thesis might seem intuitive when discussing propositional, "all-or-nothing" beliefs, Kelly (2002) and others have argued that it is implausible when beliefs are considered to be more fine-grained. For example, suppose beliefs are represented by numbers between zero and one which indicate how strongly one believes a given proposition. Jules believes that it will rain tomorrow to degree .9 , whereas Jim believes the chances are .901. The uniqueness thesis entails that at least one of Jules' or Jim's degrees of belief are irrational. Similarly, Strict Immodesty entails that Jules ought to find Jim's degrees of belief irrational and vice versa. Should the requirements of rationality really be so precise and demanding?

Many have argued so: Strict immodesty is a theorem if one's degree of belief in $\varphi$ is a probability and inaccuracy is assessed using a strictly proper scoring rule. ${ }^{8}$ However, one way of dealing with the above objection is to claim that rational belief states are never so fine-grained. Even if beliefs come in degrees and are not "all-or-nothing", it does not follow that they are fruitfully representable by single real numbers. Instead, belief might be modeled more fruitfully by indeterminate (or imprecise or "mushy") probabilities, i.e., by sets of numbers..$^{9}$ Jules and Jim both have the same inexact meteorological data that allows them to estimate the chance of rain within some margin of error. Their "real" beliefs may then be better represented by the interval $[.85, .95]$ rather than by a single real number.

Embracing indeterminate probabilities may not, on first glance, seem to help with the worry about Strict Immodesty. If Jules' belief about the probability of rain is represented by the interval $[.85, .95]$ and Jim's belief is represented by $[.8499, .95]$, Strict Immodesty entails that Jules should find Jim to be irrational, and vice versa. However, Joyce and others have argued that indeterminate probabilities are better representations of what belief states are actually warranted by inexact evidence and that they, unlike numerically precise probabilities, can handle this objection.

We turn now to consider these arguments.

\footnotetext{
${ }^{8}$ One must also assume that the set of rational belief states are those the maximize expected accuracy relative to one's degree of belief.

${ }^{9}$ The expressions "imprecise probabilities" and "indeterminate probabilities" pick out two importantly different philosophical motivations for interval-valued Bayesianism (Levi 1974), but "imprecise probabilities" (or "IP") is now used, following (Walley 1991), to refer to a broad range of models for uncertain reasoning and decision making that involve sets of probabilities (Haenni, Romeijn, Wheeler, and Williamson 2011; Augustin, Coolen, de Cooman, and Troffaes 2014; Troffaes and de Cooman 2014). See (Walley 1991, Ch. 5) and (Bradley 2014) for excellent philosophical discussions of IP.
} 


\section{IMPRECISION}

Suppose you are asked to guess whether the next ball drawn from an urn will be red. You stole a glance as the urn was filled and know that three of the ten balls deposited are red. However, the colors of the remaining seven balls are unknown to you. What probability should you assign to the proposition "The next ball pulled from urn will be red"?

A number of philosophers, including accuracy-firsters (Pettigrew 2012; Pettigrew 2014), endorse the thesis that degrees of belief ought to be constrained by objective chances. The direct inference principle (or Miller's principle, or the "Principal Principle") is a special case of such a thesis: namely, this principle claims that one's degrees of belief in an event ought to be equal to the event's objective chances when such chances are known. In the current example, you know the objective chance of drawing a red ball is at least $\frac{3}{10}$, and so it stands to reason that you ought to assign a probability no less than $\frac{3}{10}$ to that proposition. But no further information about chances is known. Does the evidence warrant a precise degree of belief greater than $\frac{3}{10}$ ? Ellsberg long ago claimed not, and Joyce agrees:

In general, a body of evidence $E_{t}$ is specific to the extent that it requires probabilistic facts to hold across all credence functions in a credal state. If $E_{t}[i s$ ] entirely specific with respect to [a proposition] $X$, then it requires [one's credence] $c(X)$ to have a single value ... [P]erfectly specific evidence produces a determinate balance of evidence for $X$. Less specific evidence leaves the balance indeterminate. When the evidence for $X$ is unspecific its credence will usually be "interval-valued," i.e., the values of $c(X) \ldots$ cover an interval $\left[x_{-}, x_{+}\right]$. It is then only determinate that the balance of evidence for $X$ is at least $x_{-}$and at most $x_{+}$. The difference between the 'upper probability' $x_{+}$and the 'lower probability' $x_{-}$provides a rough gauge of the specificity of the evidence with respect to $X$ (where smaller $=$ more specific) $($ Joyce 2005, pp.171-2).

In other words, one's beliefs ought to be as strong and no stronger than is warranted by her evidence. When exact statistical frequencies are known, an agent should assign precise probabilities to events; otherwise, her beliefs ought to be imprecise.

What is not clear from the above quotation is that, for Joyce and others (Kyburg and Pittarelli 1996; Seidenfeld, Schervish, and Kadane 2010; Wheeler 2012), imprecise degrees of belief need not always be represented by a closed convex set of probabilities. Here's an example to motivate why not. Suppose you find a coin across the street from a trick coin factory. You know that the factory produces exactly two types of coins: one lands heads one-quarter of the time and the other lands heads three-quarters of the time. How should your beliefs about the first flip of this coin be represented? It seems strange to say that your degrees of belief that the coin lands heads are represented by the closed interval $\left[\frac{1}{4}, \frac{3}{4}\right]$, as the factory does not produce coins with bias $\frac{3}{8}, \frac{1}{2}$, etc. It only produces two types of coins. So perhaps it is best to say that your degrees of belief are represented by a pair of numbers, $\left\{\frac{1}{4}, \frac{3}{4}\right\}$, which 
represents your belief that either the coin has bias $\frac{1}{4}$ or it has bias $\frac{3}{4}$ and nothing in between.

In summary, the conjunction of accuracy-first epistemology and the imprecise model of belief entails (at least) the following four theses:

A3. Imprecision: A belief states is a set of real numbers between 0 and $1 .^{10}$

A4. Quantifiability: Degrees of inaccuracy are represented by non-negative real numbers.

A5. Extensionality: For every truth-value $\omega$ and every belief state $b$, there is a single degree of inaccuracy $I(b, \omega)$ representing how inaccurate belief $b$ is. Moreover, this degree depends only upon $b$ and the truth-value $\omega$ of the proposition $\varphi$ of interest.

A6. Strict Immodesty: If an agent's belief state is $b$, then the set of rational belief states $R_{b}$ from her perspective is equal to the singleton $\{b\}$.

The careful reader will notice that the continuity assumption mentioned in the last section is missing. This is because it is not clear how to quantify the distance between beliefs if they are represented by arbitrary sets of numbers between zero and one. For example, how "close" are the beliefs that (i) the coin lands heads with some probability in the interval $\left[\frac{1}{4}, \frac{3}{4}\right]$ and (ii) the coin lands heads with some probability in the interval $\left[\frac{1}{4}, \frac{3}{4}\right]$ other than $\frac{4}{7}$ ? On first glance, it seems that an accuracy-firster who endorses imprecise probabilities would need to answer such hard questions.

However, certain cases seem unproblematic. Consider, for example, three intervalvalued belief states: $a=\left[a_{-}, a_{+}\right], b=\left[b_{-}, b_{+}\right]$, and $c=\left[c_{-}, c_{+}\right]$. Again, the intervals $a, b$, and $c$ might represent one's beliefs about whether or not a coin will land heads. Suppose that

- $a_{-} \leq b_{-} \leq c_{-}$or $a_{-} \geq b_{-} \geq c_{-}$, and

- $a_{+} \leq b_{+} \leq c_{+}$or $a_{+} \geq b_{+} \geq c_{+}$.

Then we claim that the distance between the belief states $a$ and $b$ ought to be at least as great as the distance between the belief states $a$ and $c$. Call this Constraint $\mathbf{P}$, for "Pareto". For example, suppose Alison's lower probability for some event differs from Carole's at least as much as Bill's does, in the standard way of comparing closeness of two numbers. Further, assume that Alison's upper probability for some event differs from Carole's at least as much as Bill's does. Then Constraint P entails that Alison's beliefs differ from Carole's to a degree at least as great as the degree to which Bill's and Carole's beliefs differ. Alison, Bill, and Carole's beliefs can be pictured as in the diagram below.

\footnotetext{
${ }^{10}$ In other words, the set of possible belief states $B$ is the power set of the unit interval. Our argument does not require $B$ to be the entire power set. In fact, it only needs to include all closed intervals.
} 


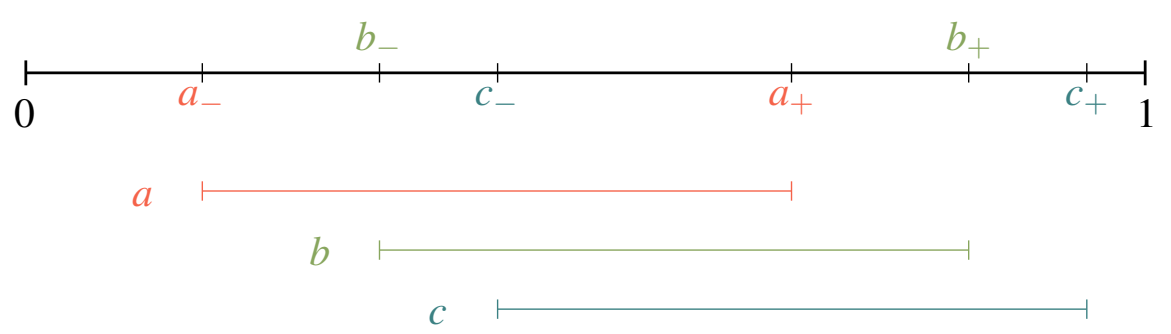

Any measure of distance between belief states that did not satisfy Constraint $\mathrm{P}$ would be bizarre. Hence, arguments for continuity of inaccuracy entail the following principle when applied to imprecise probabilistic beliefs:

A7. Continuity: Sufficiently similar belief states are similarly inaccurate. More precisely, for all $\omega$, the function $I(b, \omega)$ restricted to the set of interval beliefs $b$ is continuous with respect to the parameter $b$, where the metric on beliefs satisfies Constraint $\mathrm{P}$.

\section{IMPOSSIBILITY THEOREM}

Unfortunately, the most general version of imprecise, accuracy-first epistemology, which is based on Admissibility and Imprecision, is inconsistent.

Theorem 3.1 Admissibility, Imprecision, Continuity, Quantifiability, Extensionality, and Strict Immodesty are jointly inconsistent.

The proof of Theorem 3.1, which is in Appendix A.1, employs a mild mathematical generalization of a result due to Seidenfeld, Schervish, and Kadane (2012). Further, Theorem 3.1 extends to any finite number of propositions, but the proof requires considerably greater mathematical machinery, namely, the topological invariance of dimension. ${ }^{11}$

We turn now to consider which of these theses an accurate but imprecise epistemology should drop.

\section{A Mildly Immodest Proposal}

Accuracy-first epistemology is characterized by the conjunction of Extensionality and Admissibility. Advocates of epistemic imprecision, like Joyce, are committed to the thesis that indeterminate evidence may only warrant indeterminate degrees of beliefs. So that leaves three options for reconciling accuracy with imprecision: abandon Continuity, Quantifiability, or Strict Immodesty. ${ }^{12}$

\footnotetext{
${ }^{11}$ Thanks to Catrin Campbell-Moore for pointing this out to us.

${ }^{12}$ Readers familiar with other formal models of belief may wish to consider a fourth option: accept the claim that the specificity of one's beliefs ought to match the strength of one's evidence, but abandon Imprecision, which is the stronger thesis that specificity of belief is represented in a particular
} 
The obvious place to start is Continuity, which is an assumption that looks suspiciously like a mathematical convenience rather than a principled rationality constraint. In particular, there might be reasons for a measure of inaccuracy to contain "jumps." Perhaps it is permissible for a measure of inaccuracy to contain a discontinuity when one's indeterminate credence, $\left[x_{-}, x_{+}\right]$, shifts to a precise probability, $x$. After all, if indeterminate probabilities are intended to represent ambiguous or incomplete evidence, then a precise probability might represent the fact that an agent has significantly stronger evidence than an agent whose beliefs are interval-valued. Or perhaps measures of inaccuracy should be permitted to contain discontinuities for extreme belief states, such as when an agent adopts a precise probability of zero for a true proposition. But whatever benefits come from allowing discontinuities for these or other cases, avoiding the impossibility result is not one of them. Our proof of Theorem 3.1 shows the stronger result that a measure of inaccuracy must be discontinuous almost everywhere if the measure satisfies the remaining axioms. Formally, our proof shows there is no open set of belief states on which the inaccuracy measure can be continuous. Targeting Continuity to get around the impossibility result fails because there is no principled reason for asserting that a measure of inaccuracy ought to be almosteverywhere discontinuous.

Consider Quantifiability next. Abandoning Quantifiability may sound unintuitive, but there are principled reasons to do so. In particular, perhaps some beliefs are "infinitely" inaccurate and ought to be given the score $\infty$. The imprecise belief state $[0,1]$, for example, could be counted as infinitely inaccurate because it is completely uninformative. Or perhaps inaccuracy ought to be infinite when an agent assigns probability zero to a true proposition or assigns probability one to a false one. Just as there are plausible reasons for the inaccuracy function to be discontinuous, so too are there principle reasons that it should take infinite values.

Unfortunately, this strategy is likewise unworkable for getting around Theorem 3.1. Our proof of Theorem 3.1 actually allows the measure of inaccuracy to take infinite values. So, one cannot weaken Quantifiability by a small trick and hope to escape the impossibility result; any attempt to modify Quantifiability will demand an alternative way of representing inaccuracy that differs significantly from the use of real numbers.

In personal conversation, Joyce has indicated that he denies Quantifiability as a general thesis. According to him inaccuracy can be measured by a single real number only when degrees of belief are likewise represented by a single probability function. Alternatively, if one's credences are indeterminate, then a single number cannot (in general) be used to represent inaccuracy. Why? Here is one thought. If your degrees of belief are "indeterminate", then the distance between your degrees of belief and the truth is likewise indeterminate. So perhaps inaccuracy ought to be represented by a set of real numbers rather than by a single one. To illustrate, suppose $p$ is a precise credence and that $I(p, \omega)$ represents the inaccuracy of $p$ if $\omega$ is the truthvalue of the proposition of interest. Then it is natural to think that if one's credal

mathematical way. Instead, the reader might argue that Dempster-Shafer functions, for instance, are better models of indeterminate belief states. However, our impossibility result applies to several other formal models of belief, including Dempster-Shafer functions on finite sets. See the discussion in the appendix. 
state is represented by the interval $\left[\frac{1}{3}, \frac{2}{3}\right]$, then the inaccuracy of one's credal state is represented by the set of numbers $\left\{I(q, \omega): q \in\left[\frac{1}{3}, \frac{2}{3}\right]\right\}$ that contains the inaccuracy of every precise credence in the interval.

But if this argument for Imprecision is convincing, then one should abandon the idea that inaccuracy is numerically quantifiable at all. For just as an indeterminate credal state may have an indeterminate degree of accuracy with respect to a single proposition, so too can a precise credal state bear an indeterminate degree of accuracy to multiple propositions. If Jim strongly believes both that it rains frequently in Seattle and that Munich is in northern Germany, then his beliefs about Seattle's climate are accurate but his beliefs about German geography are not. Elsewhere, we argue that how two degrees of inaccuracy are combined into a single number depends upon one's interests and values (Mayo-Wilson and Wheeler, ms), which accuracy-firsters deny are relevant to epistemic concerns. This leaves two options: either (i) adopt the assumptions about subjective preference that are necessary for numerical representations of accuracy, which would entail Quantifiability for both precise and imprecise belief states, or (ii) abandon Quantifiability altogether. We think the first option is less damaging to Joyce's program, in which accuracy plays a central role. However, we also think that related programs in formal epistemology ought not be called "accuracyfirst", as any quantified epistemology must have some basis in subjective preference.

That leaves Strict Immodesty. Our mildly immodest proposal is that Joyce and other proponents of indeterminate probabilities should abandon Strict Immodesty as a principle of rationality, but our reasons for doing so also cast doubt on the nonpragmatic justifications given for Strict Immodesty.

In the case in which beliefs are precise probabilities, Strict Immodesty appears to be motivated by the desire to work with strictly proper scoring rules. To explain what a strictly proper scoring rule is, it is helpful to describe an empirical question raised by the traditional Dutch Book argument. In that argument, an agent's degree of belief in a particular event occurring is identified with her announced fair price, which is the price at which she would be willing both to buy and to sell a bet that is worth one unit of currency if the event occurs. In real life, bookies and gamblers do not announce fair prices: bookies sell gambles for more than they believe the gambles are worth, and gamblers are always searching for bargains. This raises the following question: is it possible to determine an individual's actual degrees of belief in the presence of strategic incentives for dishonest announcements?

De Finetti's second argument for probabilism provides an answer. Recall that in the forecasting argument agents are penalized by how far their announced degrees of belief differ from the truth. What De Finetti showed is that a strictly proper scoring rule-de Finetti used Brier's score - can be used to encourage a rational agent to announce her true degrees of belief. In other words, if the agent wishes to minimize her expected penalty, then it is uniquely rational for her to announce her actual beliefs. The uniqueness condition is crucial. If other credences also minimized expected loss, then an agent could announce beliefs other than her own with no fear of penalty. For an experimenter, strictly-proper scoring rules provide (in principle) a way to elicit honest judgments.

The traditional justification for using strictly-proper scoring rules, therefore, is 
that they are of pragmatic value to an experimenter. It would be surprising, therefore, if an independent argument established Strict Immodesty as a principle of epistemic rationality from the first-person perspective. What arguments are offered on behalf of Strict Immodesty? As it turns out, the above argument for Modesty, which appears in (Joyce 2009, p. 277), is offered in the midst of a discussion of strictly proper scoring rules. That is, Joyce's argument is offered in defense of the stronger postulate Strict Immodesty. Oddie (1997) offers the same argument for Strict Immodesty-which he calls "cogency."

A close examination of this argument, however, reveals that it does not warrant Strict Immodesty. The argument concludes that it is irrational to hold "selfundermining" credences, but even if sound, it does not follow that it is rationally required to believe that one's own beliefs are uniquely rational. Thus, we recommend mild immodesty instead:

A6*. Mild Immodesty: A rational agent always finds her own belief state to be rational, i.e., $b$ is a member of $R_{b}$.

A careful reader might ask whether there are any measures of inaccuracy satisfying the remaining postulates and Mild Immodesty; the answer is, Yes. In fact, there are infinitely many. But not all of them are intuitively compelling.

For example, imagine scoring every belief state by a lucky number, say 7 , such that $I(b, \omega)=7$ for all belief states $b$ and truth-values $\omega$. Further, suppose that for an agent in belief state $b$, the set of rational belief states $R_{b}$ from her perspective consists of those states that are at least as accurate as $b$ whatever the truth. So $R_{b}=B$, as every belief state is always accurate to the same degree. This measure of inaccuracy and way of defining rational beliefs satisfy Imprecision, Continuity, Quantifiability, Extensionality, Admissibility, and (non-strict) Immodesty. Yet no one would say $I$ is a reasonable measure of accuracy.

The question then is whether there are any plausible, mildly immodest measures of inaccuracy. What would make a measure "plausible"? Here are three obvious constraints. Consider two belief states $b$ and $c$. Suppose every precise credence in $b$ is closer to the truth-value of some proposition than is every precise credence in $c$. Then the belief state $b$ should be closer to the truth-value of the proposition than is $c$. For example, if $c=\left[\frac{1}{4}, \frac{1}{2}\right]$ and $b=\left[\frac{3}{4}, 1\right]$ are two beliefs about the probability that a coin flip lands heads, then the person who is in state $b$ unequivocally believes the coin will land heads more than does another person who is in state $c$. So if the coin does land heads, then $b$ is more accurate than $c$. If it lands tails, the opposite is true. In general, inaccuracy measures should penalize beliefs that are not truth-directed: ${ }^{13}$

A8. Truth-Directedness: Let $b, c \in B$ be any two beliefs. If $|p-\omega|<|q-\omega|$ for all precise credences $p$ in $b$ and $q$ in $c$, then $I(b, \omega)<I(c, \omega)$.

The truth-directedness axiom rules out the vacuous lucky-number measure of inaccuracy discussed above that scored all belief states equally. For if a proposition is true,

\footnotetext{
${ }^{13}$ We borrow the name "truth-directedness" from (Joyce 2009), who defends a similar criteria for precise numerical belief states.
} 
then the precise credence $b=\frac{3}{4}$ must be strictly closer to the truth than the credence $c=\frac{1}{2}$, by A7.

Nonetheless, all the postulates thus far fail to capture the sensible idea that adding bad eggs to the pan cannot improve an omelet. If Alison's degree of belief in the truth of climate change is $\frac{99}{100}$ and Bill's is $\frac{9}{10}$, then Alison's beliefs cannot be made more accurate by weakening them to the state $\left[\frac{9}{10}, \frac{99}{100}\right]$. In general, let $b, c \in B$ be two belief states such that $b$ is a subset $c$, and let $c \backslash b$ denote those credences that are in $c$ but not $b$. If every precise credence in $c \backslash b$ is strictly further from the truth than every precise credence in $b$, then $c$ should be scored no more accurate than $b$. In general, any plausible measure of inaccuracy ought to satisfy Savage's Omelet Law:

A9. SOL: Let $b, c \in B$ be two belief states such that $b \subseteq c$, and suppose that $|q-\omega|>$ $|p-\omega|$ for all $q \in c \backslash b$ and all $p \in b$. Then $I(b, \omega) \leq I(c, \omega)$.

Finally, accuracy should be monotonic: adding accurate credences cannot make a belief state less accurate. If Alison's beliefs in the truth of climate change are represented by $\left[\frac{9}{10}, \frac{99}{100}\right]$, then her belief cannot be made less accurate by weakening it to the state $\left[\frac{9}{10}, 1\right]$. In general, let $b, c \in B$ be two belief states such that $b$ is a subset $c$. If every precise credence in $c \backslash b$ is strictly closer to the truth than every precise credence in $b$, then $c$ is no less accurate than $b$.

A10. Monotonicity: Let $b, c \in B$ be two belief states such that $b \subseteq c$, and suppose that $|q-\omega| \leq|p-\omega|$ for all $q \in c \backslash b$ and all $p \in b$. Then $I(c, \omega) \leq I(b, \omega)$.

One might worry that, in order to eliminate unintuitive measures of inaccuracy, it might be necessary to continue piling on further and further constraints. Luckily, together with the above widely-accepted postulates of accuracy-first epistemology, Truth-Directedness, SOL, and Monotonicity place substantial limits on the set of acceptable mildly immodest inaccuracy measures. In fact, one can show that every mildly immodest measure of inaccuracy must satisfy a particular functional form, which we describe below in Theorem 4.1. Before stating our result, we give an example.

Suppose you wish to measure the inaccuracy of precise (probabilistic) belief states in some standard way, such as squared-error loss. How might you extend your measure to imprecise belief states? Here is one proposal: identify the inaccuracy of an imprecise belief state with the inaccuracy of its midpoint. Measuring inaccuracy by using the midpoint has some nice features. First of all, if the set of rational belief states $R_{b}$ is defined in any number of ways (e.g., as those belief states that minimize expected loss relative to the midpoint of $b$ ), then all of the above postulates are satisfied. ${ }^{14}$ Also, scoring a belief state by its midpoint is one way of formalizing the idea of scoring "average" inaccuracy of an interval-valued belief state.

On the other hand, it should be clear that this measure of inaccuracy fails to have a number of properties that one might find desirable. The midpoint of the precise credence $\left\{\frac{1}{2}\right\}$ is the same as the midpoint of the belief state $[0,1]$. So according to

\footnotetext{
${ }^{14}$ See Theorem B.4 in the Appendix.
} 
this proposed measure of inaccuracy, these two belief states will be equally accurate, regardless of the truth. Thus, measuring inaccuracy by using only one number fails to tell us how narrow or precise an agent's beliefs are.

This measure of inaccuracy is also useless from an experimenter's perspective. Recall that prior to Joyce's program, the central motivation for studying strictly-proper scoring rules was to understand how an experimenter might elicit a rational agent's degrees of belief. But if the inaccuracy of the credence $\left\{\frac{1}{2}\right\}$ is the same as that of the belief state $[0,1]$, then a rational agent has no accuracy-related incentive to report one credal state rather than another. Of course, evaluating the accuracy of an agent's beliefs and eliciting her beliefs are two different tasks, and accuracy-first epistemologists might argue that a tool for one task is appropriate for the other. Even so, surely it would be a nice feature of a measure of inaccuracy if it served both functions.

Are there any measures of inaccuracy that satisfy the above postulates and which score the accuracy of precise credences differently from imprecise credal states? The answer is, No. To state our second result, let $b$ be any belief state, which recall is a set of numbers between zero and one. Let $b_{-}$denote the greatest lower bound (i.e., infimum) of the numbers in $b$, and $b_{+}$denote the least upper bound (i.e., supremum).

TheOrem 4.1 Dominance (A2), Imprecision (A3), Quantifiability (A4), Extensionality (A5), Mild Immodesty (A6*), Continuity (A7), Truth-Directedness (A8), SOL (A9), and Monotonicity (A10) entail there is a function $f: B \rightarrow[0,1]$ such that, for any belief $b$ :

- $f(b) \in\left[b_{-}, b_{+}\right]$, and

- $I(b, \omega)=I(f(b), \omega)$ for all $\omega$

Theorem 4.1 entails that any method of measuring inaccuracy of an imprecise belief $b$ must reduce to measuring the inaccuracy of exactly one precise credence. ${ }^{15}$ This theorem, and its proof, draws on a result due to Miriam Schoenfield, who argues that there are no purely accuracy-related reasons to adopt imprecise credences rather than precise ones. ${ }^{16}$ We agree.

But we think neither the result nor the two problems discussed above are real concerns for those who are committed to Imprecision.

For Joyce's arguments for Imprecision, like those given by most imprecise probability theorists, do not appeal to considerations of accuracy. Instead, imprecision is thought to reflect the quality of evidence one has about an event. Suppose you flip a

\footnotetext{
${ }^{15}$ In the appendix, we also prove a partial converse to Theorem 4.1, which shows that virtually any strictly proper scoring rule $I^{*}$ for precise probabilities and function $f: B \rightarrow[0,1]$ can be used to define a measure of inaccuracy I on all belief states satisfying the above axioms. We note that our proof only works for measures of inaccuracy for a single proposition. We conjecture the result can be extended to finite agendas of propositions.

${ }^{16}$ Schoenfeld's theorem (Schoenfield 2015) employs different assumptions than ours, and the differences are philosophically relevant. Schoenfield's result requires somewhat strong assumptions about rationality (our function $R_{b}$ ), but it makes relatively few assumptions about the inaccuracy measure I. In contrast, our representation theorem relies on a relatively weak principle of rationality - avoid strictly dominated beliefs - but we make several assumptions about the inaccuracy measure $I$.
} 
mysterious coin scores of times and judge it to be fair. Then, you are in a different evidential position than someone who believes the coin is fair, sight unseen. In this case the quality of evidence is simply measured by the number of coin flips: more flips, better evidence. All things considered, an imprecise probability theorist might recommend that you assign $\frac{1}{2}$ to the proposition that the next flip will land heads, but counsel the other person to adopt vacuous lower and upper probabilities to heads, yielding the unit interval $[0,1]$.

A strict Bayesian will recommend assigning a precise probability of $\frac{1}{2}$ in both cases, thereby ignoring the difference between these two evidential states. Walley (1991, §5.1.5) provides a host of reasons why evidential considerations may require imprecise belief states. Examples include an agent who is completely ignorant with respect to the stochastic mechanism producing the event, such as in the coin example above; an agent who receives conflicting evidence about an event, such as when one receives contradictory testimony from different experts; an agent who places a different value on evidence about an event in the future, so that the value of evidence decreases over time. None of these arguments for Imprecision mention accuracy, nor should they.

Here is why. Suppose that Alison is a US history scholar and knows that Chester Arthur wore mutton chops. Bill, on the other hand, believes all presidents in the 19th century had mutton chops, including Arthur. Bill may be many things, but on the matter of Chester Arthur's whiskers, his belief is just as accurate as Alison's. Of course, Alison's other beliefs about the 21st President, his achievements, campaign strategies, and so on, may be more accurate than Bill's. Her beliefs may also be more reliably formed, in the sense that when new questions arise, her new beliefs are more likely to be true than Bill's. But that does not entail that her belief that Chester Arthur wore mutton chops is more accurate than Bill's. Accuracy is a static feature of belief; reliability is diachronic. ${ }^{17}$

This example suggests that, if the imprecision of a belief is determined by the strength of evidence on which it is based, then precision and accuracy may come apart. Hence, there might be many belief states, of differing levels of precision, that are equally accurate. Yet if that is true, then considerations of accuracy will generally be insufficient to narrow down the set of rational beliefs to a single state, as Strict Immodesty requires. We conclude that if the precision of a rational agent's beliefs reflects strength of evidence, then Strict Immodesty must go. So our mildly immodest proposal is motivated not only by a technical impossibility result, but also by principled considerations concerning evidence.

\footnotetext{
${ }^{17}$ One might object that Allison's total belief state is more accurate that Bill's total belief state, and so this example does not show that agents may have equally accurate beliefs but evidence of differing strengths. However, to endorse this objection, one must embrace a very strong internalist theory of evidence - stronger, even, than demanded by evidentialism (Conee and Feldman 2004). For if strength of evidence were a function of accuracy of a total belief state, then strength of evidence would be a function of belief and truth only, rather than of mental states generally.
} 
In sum our argument is this. To reconcile Joyce's commitments to accuracy and imprecision, there are two options. The first, which Joyce endorses, is to drop Quantifiability. ${ }^{18}$ Unfortunately, the case against quantifying accuracy applies whether credences are precise or imprecise. And dropping Quantifiability generally undermines existing accuracy-first arguments for probabilism, conditionalization, direct inference, and more.

In contrast, our proposal to replace Strict Immodesty with Mild Immodesty is compatible with accuracy-first arguments for many epistemic norms. Advocates of Strict Immodesty, moreover, conflate the principle with its non-strict counterpart. And the experimenter's rationale for using strictly proper scoring rules, namely, to elicit honest reports of belief, provides no reason to endorse Strict Immodesty as a principle of epistemic rationality. Finally, dropping Strict Immodesty can be justified by evidential considerations, just like Imprecision.

Our mildly immodest proposal also has broader implications. In particular, it shows that accuracy-first epistemology has substantial limits. Theorem 4.1 entails that numerical measures of accuracy cannot winnow out the class of rational beliefs alone. But this should not be a surprise. Accuracy is only one criterion for rational belief; evidential justification is another.

\section{ACKNOWLEDGEMENTS}

Thanks to Aidan Lyon for encouraging us to explore inaccuracy measures for imprecise probabilities, and for encouraging philosophical investigation of recent technical work. We are thankful as well to Teddy Seidenfeld for discussing the above impossibility result with us, and to Catrin Campbell-Moore for comments and corrections to earlier drafts. We also benefited greatly from discussions with Seamus Bradley, Jason Konek, Jim Joyce, and Richard Pettigrew about the various features of accuracy-first epistemology, and to an anonymous referee for his or her helpful suggestions. Finally, we are grateful to the Alexander von Humboldt Foundation for their generous support.

\section{A Impossibility Result}

\section{A.1 Definitions and Proof}

For any set $E$, let $2^{E}$ denote its power set. Let $\Omega$ be any set, which represents possible states of the world. Let $\mathscr{A} \subseteq 2^{\Omega}$ be a collection of subsets of $\Omega$. Elements of $\mathscr{A}$ represent propositions, and $\mathscr{A}$ represents the agenda of interest. Let BEL be any

\footnotetext{
${ }^{18}$ Seidenfeld et. al. (2012) also endorse dropping quantifiability for the purpose of eliciting rational agents' beliefs.
} 
topological space. Members of BEL represent belief states. Let $\langle S, \preceq\rangle$ be a partiallyordered set; elements of $S$ represent inaccuracy scores. An inaccuracy measure for the agenda $\mathscr{A}$ is a function $I_{\mathscr{A}}:$ BEL $\times \Omega \rightarrow S$. Let $T_{\mathscr{A}}: \Omega \rightarrow 2^{\mathscr{A}}$ be the map $T_{\mathscr{A}}(\omega)=$ $\{A \in \mathscr{A}: \omega \in A\}$. The function $T$ represents a truth-evaluation, in the sense that $T_{\mathscr{A}}(\omega)$ is equal to the set of propositions true in state of the world $\omega$. Finally, let $R$ : $\mathrm{BEL} \rightarrow 2^{\mathrm{BEL}}$ be a function. $R_{b}=R(b)$ represents the set of beliefs that are considered rational by a person with beliefs $b$.

We now provide formal statements of axioms in the body of the paper. An inaccuracy measure $I_{\mathscr{A}}$ is called extensional if $I_{\mathscr{A}}(b, \omega)=I_{\mathscr{A}}\left(b, \omega^{\prime}\right)$ whenever $T_{\mathscr{A}}(\omega)=$ $T_{\mathscr{A}}\left(\omega^{\prime}\right)$. It is quantifiable if $S$ is equal to $\mathbb{R} \cup\{-\infty, \infty\}$ with the standard ordering. If $S$ is also a topological space, then we say $I_{\mathscr{A}}$ is continuous if $I_{\mathscr{A}}(\cdot, \omega)$ is continuous for every $\omega \in \Omega$. The function $R$ is called immodest if $b \in R_{b}$ for all beliefs $b$, and it is strictly immodest if $R_{b}=\{b\}$. Say $c$ is $I_{\mathscr{A}}$-admissible with respect to $b$ if $I_{\mathscr{A}}(c, \omega) \leq I_{\mathscr{A}}(b, \omega)$ for all $\omega \in \Omega$, and $c$ say $I_{\mathscr{A}}$-dominates $b$ if $I_{\mathscr{A}}(c, \omega)<I_{\mathscr{A}}(b, \omega)$ for all $\omega \in \Omega$. The map $R$ respects $I_{\mathscr{A}}$-admissibility if for all beliefs $b, c, d \in \mathrm{BEL}$, it follows that $c \in R_{d}$ if $b \in R_{d}$ and $c$ is $I_{\mathscr{A}}$-admissible with respect to $b$. The map $R$ respects $I_{\mathscr{A}}$-dominance if $b \notin R_{d}$ if $b$ is $I_{\mathscr{A}}$-dominated by $c$.

If $I_{\mathscr{A}}$ is extensional, then there are functions $i_{\alpha}:$ BEL $\rightarrow S$ indexed by $\alpha \in 2^{\mathscr{A}}$ such that $I(b, \omega)=i_{\alpha}(b)$ whenever $T_{\mathscr{A}}(\omega)=\alpha$. If $\mathscr{A}$ has cardinality $n \in \mathbb{N}$, then we enumerate these functions $i_{0}, i_{1}, \ldots i_{2^{n}-1}$. So in the special case in which $\mathscr{A}=\{A\}$ contains only one proposition, there are functions $i_{0}, i_{1}:$ BEL $\rightarrow S$ such that

$$
I_{\mathscr{A}}(b, \omega)=\left\{\begin{array}{l}
i_{0}(b) \text { if } \omega \notin A \\
i_{1}(b) \text { if } \omega \in A
\end{array}\right.
$$

Thus, if $I_{\mathscr{A}}$ is extensional, then $c$ is $I_{\mathscr{A}}$-admissible with respect to $b$ if $i_{k}(c) \leq i_{k}(b)$ for all $k<2^{n}$. Similarly, if if $I_{\mathscr{A}}$ is extensional and continuous, then $i_{k}$ is continuous for all $k<2^{n}$.

Lemma A.1 Suppose $I_{\mathscr{A}}$ is extensional and $\mathscr{A}$ has finite cardinality $n$. Consider the map $\mathbf{I}_{\mathscr{A}}^{-j}: \mathrm{BEL} \rightarrow S$ defined by $b \mapsto\left\langle i_{0}(b), \ldots i_{j-1}(b), i_{j+1}(b), \ldots, i_{2^{n}-1}(b)\right\rangle$. If $R$ is strictly immodest and respects $I_{\mathscr{A}}$-admissibility, then $\mathbf{I}_{\mathscr{A}}^{-j}$ is injective. It follows that if $\mathscr{A}=\{A\}$, both $i_{0}$ and $i_{1}$ are injective.

Proof. Suppose $\mathbf{I}_{\mathscr{A}}^{-j}(b)=\mathbf{I}_{\mathscr{A}}^{-j}(c)$, or in other words, that $i_{k}(b)=i_{k}(c)$ for all $k \neq j$. Either $i_{j}(b) \preceq i_{j}(c)$ or $i_{j}(c) \preceq i_{j}(b)$ (or both). Without loss of generality, assume $i_{j}(b) \preceq i_{j}(c)$. Then $b$ is $I_{\mathscr{A}}$-admissible with respect to $c$. Because $R$ is strictly immodest, $R_{c}=\{c\}$. As $R$ respects $I_{\mathscr{A}}$-admissibility and $b$ is $I_{\mathscr{A}}$-admissible with respect to $c$, it follows that $b \in R_{c}=\{c\}$. Thus, $b=c$.

Before stating the impossibility theorem, we review one basic fact from topology:

LEMMA A.2 Let $V$ be any connected subset of a topological space, and suppose $V$ contains three distinct points $v_{1}, v_{2}$, and $v_{3}$ such that $V \backslash\left\{v_{1}, v_{2}, v_{3}\right\}$ is also connected. Then there is no continuous injection $f: V \rightarrow \mathbb{R} \cup\{-\infty,+\infty\}$.

THEOREM A.3 Let $\tau$ denote the topology on BEL. Assume: 
1. $\mathscr{A}$ is a singleton,

2. Every non-empty open set in $\tau$ contains a connected subset $V$ and three points $v_{1}, v_{2}, v_{3} \in V$ such that $V \backslash\left\{v_{1}, v_{2}, v_{3}\right\}$ is also connected,

3. $I_{\mathscr{A}}$ is extensional and quantifiable, and

4. $R$ is strictly immodest and respects $I_{\mathscr{A}}$-admissibility.

Let $\tau^{*}$ be any topology that is at least as fine as $\tau$. For both $n=0$ and $n=1$, there is no non-empty open set $U \in \tau$ such that the restriction of $i_{n}$ to $U$ is continuous with respect to $\tau^{*}$.

Proof. By contradiction. Assume $i_{n}$ is continuous with respect to $\tau^{*}$ on a non-empty open set $U \in \tau$. As $\tau^{*}$ is at least as fine as $\tau$, it follows that $i_{n}$ is continuous with respect to $\tau$. By the second assumption, $U$ contains a connected subset $V$ and three points $v_{1}, v_{2}, v_{3} \in V$ such that $V \backslash\left\{v_{1}, v_{2}, v_{3}\right\}$ is also connected. As $i_{n}$ is continuous with respect to $\tau$, the restriction of $i_{n}$ to $V$ is also continuous respect to $\tau$. By lemma A.1 and the third and forth conditions, $i_{n}$ is injective. Since $I_{\mathscr{A}}$ is quantifiable, it follows that $i_{n}$ is a continuous injection from $V$ to $\mathbb{R} \cup\{-\infty, \infty\}$, contradicting Lemma A.2

The next four examples show how Theorem A.3 applies to several different models of belief, including interval-valued probabilities, ranking functions, and DempsterShafer belief functions.

Example 1: Let BEL $=\mathscr{B}$ be any subset of the power set of the unit interval such that $\mathscr{B}$ contains all closed intervals $[x, y] \subseteq[0,1]$. The set $\mathscr{B}$ will be called the set of imprecise beliefs. Let $\mathscr{C} \subseteq \mathscr{B}$ be the set of all closed interval-valued beliefs, which we will identify with the set $\{\langle p, q\rangle \in[0,1] \times[0,1]: p \leq q\}$ via that mapping $[p, q] \mapsto\langle p, q\rangle$. Let $\mathscr{P}=\{[p, p]: p \in[0,1]\} \subseteq \mathscr{C}$ be the set of precise credences. Consider any metric $m$ on $\mathscr{B}$ satisfying Constraint P in Section 2. By Lemma A.4 below, the topology induced by $m$ on $\mathscr{C}$ is finer than the Euclidean topology. Further, any open ball $V$ in $n \geq 2$ dimensional Euclidean space is connected, and upon removing any three points from such a ball, one obtains a set that is connected (in fact, path-connected). Hence, by Theorem A.3, there is no inaccuracy score $I_{\mathscr{A}}$ and no function $R$ for an agenda of one propositions $\mathscr{A}$ such that both (1) $I_{\mathscr{A}}$ is extensional, quantifiable, and continuous, and (2) $R$ is strictly immodest and $I_{\mathscr{A}}$-admissible.

Example 2: Let $B, D$, and $E$ be three disjoint sets, which each contain at least four elements. Members of $B$ represent differing strengths of belief in a proposition; $D$ represents strengths of disbelief; and $E$ represents different ways of "suspending judgment" (e.g., by feeling more confident in a proposition than its negation, but not enough to believe the proposition). Let BEL $=B \cup D \cup E$, and let $\mathscr{V}=\{B, D, E\}$ be a partition of BEL. Then $\mathscr{V}$ is clearly a basis for a topology, and so we can let $\tau$ be the (quotient) topology on BEL generated by $\mathscr{V}$. Notice that BEL is not metrizable, as $\mathscr{V}$ is not Hausdorff. Each of the three sets is clearly connected and because each element of $V \in \mathscr{V}$ 
contains at least four elements, it follows that each $V$ contains three points $v_{1}, v_{2}, v_{3}$ such that $V \backslash\left\{v_{1}, v_{2}, v_{3}\right\}$ is connected. As every non-empty open set is a non-empty union of $B, D$, and $E$, it follows that BEL satisfies the assumption of Theorem A.3.

Example 3: Let $\mathscr{F}$ be an algebra over $\Omega$. A ranking function is a map $b: \mathscr{F} \rightarrow$ $\mathbb{R} \cup\{\infty\}$ such that $b(\emptyset)=\infty, b(\Omega)=0$, and $b(E \cup F)=\min \{b(E), b(F)\}$. The value $b(E)$ is intended to represent the degree to which the proposition $E$ is disbelieved. For simplicity, assume that $\mathscr{F}=2^{\Omega}$ and that $\Omega=\left\{\omega_{1}, \ldots, \omega_{n}\right\}$ is finite, where $n>2$. Then it is easy to show, by induction on the size of $E$, that $b(E)=\min \{b(\{\omega\}): \omega \in E\}$. Hence, for each ranking function $b$, there is some $\omega_{i}$ such that $b\left(\left\{\omega_{i}\right\}\right)=0$. Define an equivalence relation $\equiv$ over ranking functions such that $b \equiv c$ precisely when two conditions hold: first, $b(E)=\infty$ if and only if $c(E)=\infty$, and second, $b(E)=0$ if and only if $c(E)=0$. Let $[b]$ denote the equivalence class of $b$. For any $b$, let $I_{b} \subset\{1, \ldots, n\}$ be the set of $i$ such that $b\left(\left\{\omega_{i}\right\}\right) \notin\{0, \infty\}$. Hence if $I_{b} \neq \emptyset$, then any ranking function $c \in[b]$ can be identified with a point in Euclidean space, namely, $\left\langle c\left(\left\{\omega_{i}\right\}\right)\right\rangle_{i \in I_{b}}$.

Let BEL be the set of ranking functions such that $\left|I_{b}\right| \geq 2$. For any two beliefs $b$ and $c$, if $b \equiv c$, then let $e(b, c)$ be the Euclidean distance between $b$ and $c$ when considered as points in Euclidean space. Consider the collection of sets $\mathscr{U}=\left\{B_{\mathcal{\varepsilon}}^{m}(b)\right.$ : $b \in \mathrm{BEL}, \varepsilon>0\}$, where $B_{\varepsilon}^{m}(b)=\{c \in[b]: m(b, c)<\varepsilon\}$. It is easy to show that $\mathscr{U}$ is a basis for a topology $\tau$ on beliefs, and moreover, each $B_{\varepsilon}^{m}(b)$ is homeomorphic to an open ball in the subpace topology of $\left(\mathbb{R}^{\left|I_{b}\right|}\right)^{+}=\left\{r \in \mathbb{R}^{\left|I_{b}\right|}: r_{i}>0\right.$ for all $i \leq$ $\left.\left|I_{b}\right|\right\}$. Thus, since each open set in $\tau$ contains a set homeomorphic to an open ball in Euclidean space dimension greater than two, it follows that each open set in $\tau$ contains a connected set from which three points can be removed to obtain another connected set. So Theorem A.3 applies.

Example 4: A Dempster-Shafer belief function is a map $b: 2^{\Omega} \rightarrow[0,1]$ such that

- $b(\emptyset)=0$,

- $\sum_{A \subseteq \Omega} b(A)=1$, and

- $b\left(\bigcup_{j \leq n} A_{j}\right) \geq \sum_{I \subseteq\{1,2, \ldots n\}}(-1)^{|I|+1} b\left(\bigcap_{i \in I} A_{i}\right)$.

A mass function is a map $m: 2^{\Omega} \rightarrow[0,1]$ such that $m(\emptyset)=0$ and $\sum_{A \subseteq \Omega} m(A)=1$. It is well-known that, if $\Omega$ is finite, then for any Dempster-Shafer function $b$, there is a unique mass function $m_{b}$ such that $b(A)=\sum_{C \subseteq A} m_{b}(C)$. Thus, if $\Omega=\left\{\omega_{1}, \ldots, \omega_{n}\right\}$ is finite, then any Demspter-Shafer function $b$ can be represented by a point in $\left(2^{n}-\right.$ $2)$-dimensional Euclidean space, namely, $\left\langle m_{b}(A)\right\rangle_{\emptyset \subset A \subset \Omega}$. Any metric on Euclidean space, therefore, is also a metric over Dempster-Shafer functions. Call a mass function regular if $m(A)>0$ for any non-empty set $A$. Suppose that $\Omega$ contains at least two points, and let BEL be the set of Dempster-Shafer belief functions generated by regular mass functions. Consider any metric over $\mathbb{R}^{|\Omega|}$ that induces a topology no coarser than the Euclidean topology. Then the Theorem A.3 applies. 
The following lemma is used in Example 1 above.

Lemma A.4 Let $m$ be any metric on $\mathscr{B}$ satisfying Constraint $P$ in Section 2. The topology induced by $m$ on $\mathscr{C}$ is finer than the Euclidean topology.

Proof: It suffices to show that every open ball in the Euclidean metric contains an open ball with respect to $d$. To do so, fix $b=\left\langle b_{1}, b_{2}\right\rangle \in \mathscr{C}$. Let $\varepsilon>0$ and $B_{\varepsilon}^{e}(b)$ be an open ball around $b$ in the Euclidean metric $e$. We must find some $\delta>0$ such that $B_{\delta}^{m}(b) \subseteq B_{\mathcal{\varepsilon}}^{e}(b)$, where $B_{\delta}^{m}(b)$ is a ball around $b$ with respect to the metric $m$. We claim $\delta=\inf \left\{m(b, c): c \in \mathbb{R}^{2}\right.$ and $\left.e(b, c)=\varepsilon\right\}$ does the job. First, we must show that $\delta>0$. Define:

$$
\begin{array}{ll}
u=\left\langle b_{1}, b_{2}+\frac{\sqrt{2} \varepsilon}{2}\right\rangle, \quad l=\left\langle b_{1}, b_{2}-\frac{\sqrt{2} \varepsilon}{2}\right\rangle \\
d=\left\langle b_{1}-\frac{\sqrt{2} \varepsilon}{2}, b_{2}\right\rangle, \quad r=\left\langle b_{1}+\frac{\sqrt{2} \varepsilon}{2}, b_{2}\right\rangle
\end{array}
$$

Let $\beta=\min \{m(b, u), m(b, d), m(b, l), m(b, r)\}$.

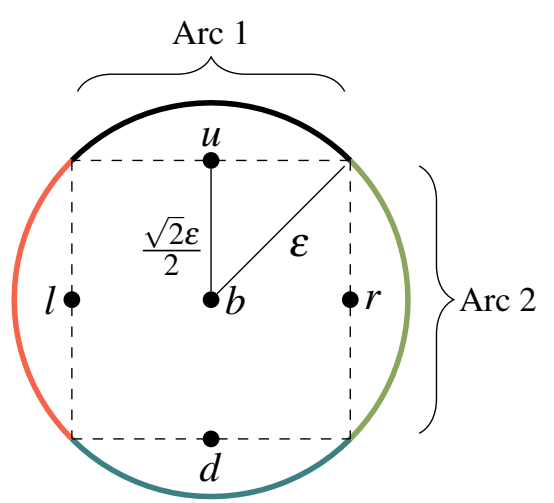

Figure 1 As $m$ is a metric, each element of the set $\{m(b, u), m(b, d), m(b, l), m(b, r)\}$ is positive, and hence, so is $\beta$. Consider Figure 1. By Constraint $\mathrm{P}$, each point $c$ on Arc 1(in black) above $u$ is at least as far with respect to $m$ from $b$ than is the point $u$. Similarly, each point on the Arc 2 (in green) to the right of $r$ is at least as far with respect to $m$ from $b$ than is $r$. Similarly for the two arcs below $u$ and to the left of $l$, respectively. It follows that $0<\beta \leq m(b, c)$ for all $c \in \mathbb{R}^{2}$ such that $m(b, c)=\varepsilon$. By definition of $\delta$, it follows that $0<\beta \leq \delta$.

Now we show that $B_{\delta}^{m}(b) \subseteq B_{\varepsilon}^{e}(b)$. To do so, let $c=\left\langle c_{1}, c_{2}\right\rangle \notin B_{\varepsilon}^{e}(b)$. Let $f=$ $\left\langle f_{1}, f_{2}\right\rangle$ be the projection of $c$ onto the closed ball (with respect to the Euclidean metric) of radius $\varepsilon$ around $b$. (See Figure 2.) Note that, if $c$ itself lies on the closed ball of radius $\varepsilon$, then $f=c$. Regardless, $\left|b_{1}-f_{1}\right| \leq\left|b_{1}-c_{1}\right|$ and $\left|b_{2}-f_{2}\right| \leq\left|b_{2}-c_{2}\right|$. So by Constraint $\mathrm{P}$, it follows that $m(b, f) \leq m(b, c)$. By definition of $\delta$, we know that $\delta \leq m(b, f)$. Hence, $m(b, c) \geq \delta$ and $c \notin B_{\delta}^{m}(b)$.

\section{Discussion of the Result}

Theorem A.3 is a modest mathematical generalization of Seidenfeld, Schervish, and Kadane's (2012) Proposition 5. There are three philosophical reasons to state the result in our greater generality.

First, as stated in Seidenfeld, Schervish, and Kadane's (2012), the decision rule $R$ must reduce to expected-utility maximization when the belief state is a precise probability. The above proof shows that this restriction is unnecessary. This is important because philosophical objections to expected utility-maximization provide no reason 
to believe there are inaccuracy measures that are strictly immodest. As long as rationality requires that agents obey a very weak, pairwise admissibility assumption as above, there are no way to establish a strictly immodest inaccuracy measure.

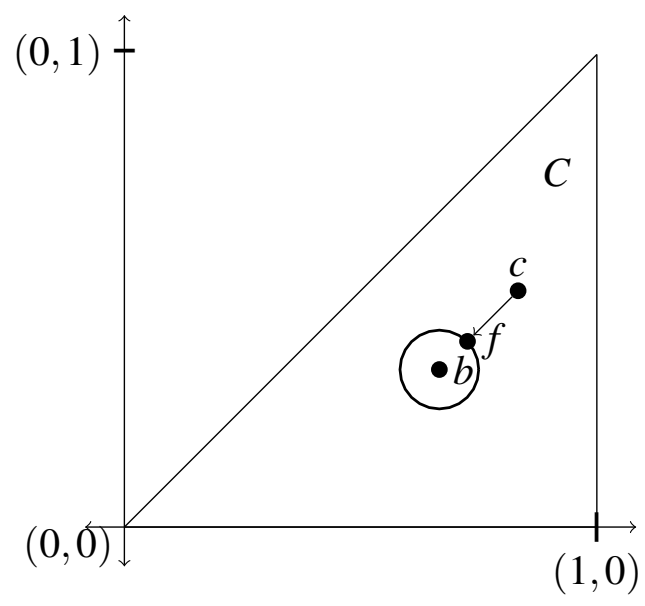

Figure 2

Second, Seidenfeld, Schervish, and Kadane's Proposition 5 states that there is no continuous strictly proper scoring rule for imprecise probability forecasts. So every strictly proper scoring rule must be discontinuous somewhere. Hence, on first glance, this leaves open the possibility that there is a strictly proper scoring rule that is continuous on a large subset of forecasts. However, the above argument shows that if $I$ is strictly immodest, then it is discontinuous on a dense subset of forecasts. This is important for the reasons discussed in the body of the paper.

Third, Proposition 5 (Seidenfeld, Schervish, and Kadane 2012) is restricted to real-valued scoring rules; the argument above shows this is unnecessary, and the plausibility of scoring rules taking infinite extended-real values is discussed in the text.

Finally, Seidenfeld, Schervish, and Kadane restrict their attention to interval-valued imprecise probabilities. Theorem 3.1 indicates that their proof strategy also applies to a wider class of imprecise belief states, which may include non-convex sets of real numbers. In this wider context, only mild restrictions (Constraint P) on the metric quantifying distance between belief states are necessary in order to generate the same impossibility result. In fact, because the main step in the proof (the employment of Lemma A.2) relies only on topological properties, the theorem also applies to other formal models of belief, including ones in which the set of belief states is non-metrizable, as shown above in Example 2.

In personal correspondence, Catrin Campbell-Moore has shown us another important way in which Seidenfeld, Schervish, and Kadane's Proposition 5 can be generalized. Namely, a similar proof holds if $\mathscr{A}$ contains more than one proposition. In this case, then the proof of Lemma A.4 generalizes in the obvious way. However, the use of Lemma A.2 in the proof of Theorem A.3 must be replaced by the use of a more powerful result, such as the invariance of dimension theorem, which shows there is no continuous injection from a non-empty, open subset $U \subseteq \mathbb{R}^{m}$ to $\mathbb{R}^{n}$ if $m>n$. Employing the invariance of dimension theorem compromises a bit of topological generality, but some theorem like it is necessary for agendas with more than one proposition. Moreover, because several of the most important formal models of belief (e.g., imprecise credences and Dempster-Shafer functions) are embeddable in Euclidean space, the impossibility result for higher dimensions is still significant, even if it may be a bit less topologically general. 


\section{B Representation TheOREM}

In this section, we assume $\mathscr{A}$ contains exactly one proposition. We also restrict attention to the case in which BEL is equal to the set of imprecise credences $\mathscr{B}$ as described in Example 1 in the previous section. When $p$ is a precise credence, we write $I(p, \omega)$ instead of $I(\{p\}, \omega)$, and similarly, we write $i_{n}(p)$ instead of $i_{n}(\{p\})$.

LEMMA B.1 Dominance (A2) and Mild Immodesty $\left(A 6^{*}\right)$ entail that there every belief state $b \in \mathrm{BEL}$ is undominated.

Proof. If $c$ were to dominate $b$, then by Dominance $b \notin R_{b}$, contradicting Mild Immodesty.

Lemma B.2 Let $b \in \mathscr{B}$ be given, and let $b_{-}=\inf b$ and $b_{+}=\sup b$. Quantifiability (A4), Extensionality (A5), Truth-Directedness (A8), SOL (A9), and Monotonicity (A10) entail that

1. $i_{0}\left(b_{-}\right) \leq i_{0}(b) \leq i_{0}\left(b_{+}\right)$, and

2. $i_{1}\left(b_{+}\right) \leq i_{1}(b) \leq i_{1}\left(b_{-}\right)$.

Proof. If $b_{-} \notin b$, then A8 entails that $i_{0}\left(b_{-}\right)<i_{0}(b)$. If $b_{-} \in b$, then A9 entails that $i_{0}\left(b_{-}\right) \leq i_{0}(b)$. Similarly, if $b_{+} \notin b$, then A8 entails that $i_{0}(b)<i_{0}\left(b_{+}\right)$, and if $b_{+} \in b$, then A10 entails that $i_{0}(b) \leq i_{0}\left(b_{+}\right)$. The second statement is proven similarly.

Theorem B.3 Dominance (A2), Quantifiability (A4), Extensionality (A5), Mild Immodesty (A6*), Continuity (A7), Truth-Directedness (A8), and SOL (A9) entail there is a function $f: \mathscr{B} \rightarrow[0,1]$ such that, for any belief $b$ :

- $f(b) \in\left[b_{-}, b_{+}\right]$, and

- $I_{\mathscr{A}}(b, \omega)=I_{\mathscr{A}}(f(b), \omega)$ for all $\omega$

Proof. Let $b$ be any fixed belief state. By Lemma B.2, we know that $i_{0}\left(b_{-}\right) \leq i_{0}(b) \leq$ $i_{0}\left(b_{+}\right)$. Consider the function $i_{0}$ restricted to $\left[b_{-}, b_{+}\right]$. As $i_{0}$ is continuous, the intermediate value theorem entails that there is some $p \in\left[b_{-}, b_{+}\right]$such that $i_{0}(b)=i_{0}(p)$. By symmetric reasoning, there is $q \in\left[b_{-}, b_{+}\right]$such that $i_{1}(b)=i_{1}(q)$. We claim $p=q$.

Suppose for the sake of contradiction that $p<q$. Pick $r \in(p, q)$. Then by A8:

$$
\begin{aligned}
& i_{0}(b)=i_{0}(p)<i_{0}(r) \text { and } \\
& i_{1}(b)=i_{1}(q)<i_{1}(r)
\end{aligned}
$$

So $b$ dominates $r$, contradicting Lemma B.1. If $q<p$, pick $r \in(q, p)$. Then by A8:

$$
\begin{aligned}
& i_{0}(r)<i_{0}(p)=i_{0}(b) \text { and } \\
& i_{1}(r)<i_{1}(q)=i_{1}(b)
\end{aligned}
$$

So $r$ dominates $b$, contradicting Lemma B.1. Hence, $p=q$. As $b$ was arbitrary, we can define the function $f: \mathscr{B} \rightarrow[0,1]$ by $b \mapsto p$, and we're done. 
As it stands, Theorem B.3 is not actually a representation theorem: it's missing a converse theorem that asserts that any measure of inaccuracy $I_{\mathscr{A}}$ (together with a definition of $R_{b}$ ) having the particular functional form $I_{\mathscr{A}}(b, \omega)=I_{\mathscr{A}}(f(b), \omega)$ satisfies the axioms A1-A6* and A7-A10 if $f$ satisfies certain constraints. Before stating the next theorem, we state a restricted version of A8 for precise credences, namely, we say $I_{\mathscr{A}}^{*}: \mathscr{P} \times \Omega \rightarrow \mathbb{R}$ satisfies A8 if $|p-n|>|q-n|$ entails that $I_{\mathscr{A}}^{*}(p, \omega)>I_{\mathscr{A}}^{*}(q, \omega)$, where $n=1$ if $\omega \in A$ and $n=0$ otherwise.

TheOREM B.4 Suppose $I_{\mathscr{A}}^{*}: \mathscr{P} \times \Omega \rightarrow \mathbb{R}$ is extensional, continuous, and satisfies A8. Let $f: \mathscr{C} \rightarrow[0,1]$ be any continuous function (with respect to $m$ ) satisfying the following constraints: for all $b, c \in \mathscr{B}$

1. $f(b) \in\left[b_{-}, b_{+}\right]$,

2. If $p<q$ for all $p \in b$ and $q \in c$, then $f(b)<f(c)$,

3. If $b \subseteq c$ and $p<q$ for all $p \in b$ and $q \in c \backslash b$, then $f(b) \leq f(c)$, and

4. If $b \subseteq c$ and $q<p$ for all $p \in b$ and $q \in c \backslash b$, then $f(c) \leq f(b)$.

Let $I_{\mathscr{A}}(b, \omega)=I_{\mathscr{A}}^{*}(f(b), \omega)$ and $R_{b}=\left\{c \in \mathscr{B}: f(c) \in\left[b_{-}, b_{+}\right]\right\}$for all $b$. Then the triple $\langle\mathscr{B}, I, R\rangle$ satisfies $A 1-A 6^{*}$ and $A 7-A 10$.

Proof. A3-A5 are trivial. Dominance (A2) and Admissibility (A1) follow from the definition of $R$. Mild Immodesty (A $\left.6^{*}\right)$ follows from the first restriction on $f$. A8A10 follow from the second through fourth conditions and the assumption that $I_{\mathscr{A}}^{*}$ satisfies A8. The continuity of $I_{\mathscr{A}},(\mathrm{A} 7)$, follows from the continuity of $I_{\mathscr{A}}^{*}$ and $f$.

\section{REFERENCES}

Augustin, T., F. P. A. Coolen, G. de Cooman, and M. C. M. Troffaes (2014). Introduction to Imprecise Probabilities. Chichester, West Sussex: Wiley and Sons.

Bradley, S. (2014). Imprecise probabilities. In E. N. Zalta (Ed.), The Stanford Encyclopedia of Philosophy (Winter 2014 ed.).

Christensen, D. (1996). Dutch-book arguments depragmatized: Epistemic consistency for partial believers. Journal of Philosophy 93, 450-479.

Conee, E. and R. Feldman (2004). Evidentialism. Oxford: Oxford University Press.

D'Agostino, M. and C. Sinigaglia (2010). Epistemic accuracy and subjective probability. In M. Suárez, M. Dorato, and M. Rédei (Eds.), EPSA Epistemology and Methodology of Science, pp. 95-105. Springer.

de Finetti, B. (1937). La prévision: ses lois logiques, ses sources subjectives. Annales de l'Institut Henri Poincaré 7(1), 1-68.

de Finetti, B. (1974). Theory of Probability (1990 ed.), Volume I. New York: John Wiley. 
de Finetti, B. (1981). The role of Dutch books and proper scoring rules. The British Journal for the Philosophy of Science 32, 55-56.

Easwaran, K. and B. Fitelson (2012). An 'evidentialist' worry about Joyce's argument for probabilism. Dialectica 66(3), 425-433.

Feldman, R. (2011). Reasonable religious disagreements. In A. Goldman and D. Whitcomb (Eds.), Social Epistemology: Essential Readings, pp. 137-158. Oxford University Press.

Fishburn, P. C. (1970). Utility Theory for Decision Making. New York: Wiley and Sons.

Gibbard, A. (2007). Rational credences and the value of truth. In T. S. Gendler and J. Hawthorne (Eds.), Oxford Studies in Epistemology, Volume 2, pp. 143-164. Oxford University Press.

Greaves, H. and D. Wallace (2006). Justifying conditionalization: Conditionalization maximizes expected epistemic utility. Mind 115, 607-632.

Haenni, R., J.-W. Romeijn, G. Wheeler, and J. Williamson (2011). Probabilistic Logics and Probabilistic Networks. Synthese Library. Dordrecht: Springer.

Joyce, J. M. (1998). A nonpragmatic vindication of probabilism. Philosophy of Science 65(4), 575-603.

Joyce, J. M. (2005). How probabilities reflect evidence. Philosophical Perspectives 19(1), 153-178.

Joyce, J. M. (2009). Accuracy and coherence: Prospects for an alethic epistemology of partial belief. In Degrees of belief, pp. 263-297. Springer.

Joyce, J. M. (2010). A defense of imprecise credences in inference and decision making. Philosophical Perspectives 24(1), 281-323.

Kelly, T. (2002). The rationality of belief and some other propositional attitudes. Philosophical Studies 110, 163-196.

Kyburg, Jr., H. E. and M. Pittarelli (1996). Set-based Bayesianism. IEEE Transactions on Systems, Man and Cybernetics A 26(3), 324-339.

Leitgeb, H. and R. Pettigrew (2010). An objective justification of Bayesianism I: Measuring accuracy. Philosophy of Science 77(2), 201-235.

Levi, I. (1974). On indeterminate probabilities. Journal of Philosophy 71, 391-418.

Mayo-Wilson, C. and G. Wheeler (2015). Epistemic decision theory's reckoning. Unpublished manuscript.

Oddie, G. (1997). Conditionalization, cogency, and cognitive value. The British Journal for the Philosophy of Science 48, 533-41.

Pettigrew, R. (2012). Accuracy, chance, and the principal principle. Philosophical Review 121(2), 241-275.

Pettigrew, R. (2013). Accuracy and evidence. Dialectica 67(4), 579-596. 
Pettigrew, R. (2014). Accuracy, risk, and the principle of indifference. Philosophy and Phenomenological Research.

Ramsey, F. P. (1926). Truth and probability. In R. B. Braithwaite (Ed.), The Foundations of Mathematics and Other Logical Essays, pp. 156-198, 1931. London: Kegan, Paul, Trench \& Company.

Savage, L. J. (1972). Foundations of Statistics. New York: Dover.

Schoenfield, M. (2015). The accuracy and rationality of imprecise credences. Noûs.

Seidenfeld, T., M. J. Schervish, and J. B. Kadane (2010). Coherent choice functions under uncertainty. Synthese 172(1), 157-176.

Seidenfeld, T., M. J. Schervish, and J. B. Kadane (2012). Forecasting with imprecise probabilities. International Journal of Approximate Reasoning 53(8), $1248-1261$.

Sen, A. K. (1971). Choice functions and revealed preference. The Review of Economic Studies 38(3), 307-317.

Troffaes, M. C. M. and G. de Cooman (2014). Lower Previsions. Chichester, West Sussex: Wiley and Sons.

Walley, P. (1991). Statistical Reasoning with Imprecise Probabilities. London: Chapman and Hall.

Wheeler, G. (2012). Objective Bayesianism and the problem of non-convex evidence. The British Journal for the Philosophy of Science 63(3), 841-50.

White, R. (2005). Epistemic permissiveness. Philosophical perspectives 19(1), $445-459$. 